\title{
Una introducción a los teoremas de punto fijo y a la existencia de equilibrios en economía
}

An introduction to fixed point theorems and existence of equilibrium in economics

\author{
La meta es lograr una comprensión genuina de las matemáticas como un todo \\ interconectado y como una base para el pensamiento y acción científicos. \\ Richard Courant y Herbert Robbins, 2010
}

Gabriel Delgado Toral ${ }^{*}$

\section{Resumen}

El siguiente documento describe en qué consisten los teoremas de punto fijo y cómo se insertan en algunas nociones de equilibrio en economía. En la primera sección se define qué es un punto fijo de una función y de una correspondencia, particularmente los teoremas de punto fijo de Brouwer y de Kakutani y qué es un equilibrio; la segunda revisa el equilibrio walrasiano y el equilibrio de Nash, explicitando los teoremas de punto fijo para demostrar su existencia. Los comentarios finales dan cuenta de que las técnicas de modelación se han extendido casi a la par con nuevos supuestos en la profesión, que revolucionaron nuestra manera de estudiar economía.

\section{Palabras clave:}

- Metodología en economía

- Teoremas de punto fijo

- Equilibrio general

- Equilibrio de Nash

\section{Abstract}

The following document describes what constitutes fixed point theorems and how they fit into some notions of equilibrium in economics. The first section defines fixed points for functions and for correspondence, particularly the Brouwer's and Kakutani's fixed point theorems and what is an equilibrium; the second reviews the walrasian equilibrium and the Nash equilibrium, showing the relevance of the fixed point theorems to prove its existence. Concluding remarks realize that modeling techniques have spread almost on par with new assumptions in the profession, which revolutionized the way we study economics.

\section{Keywords:}

- Economic methodology

- Fixed point theorems

- General equilibrium

- Nash equilibrium

JEL: B41, C62, C70, D50

En la mayoría de las ciencias, tanto las naturales como las sociales, la formulación de modelos es esencial para explicar determinados fenómenos relevantes. La economía, como es sólito, no es ajena a esa práctica. Los economistas, independientemente del marco teórico-conceptual-metodológico que los arrope, buscan en todo momento hacer abstracciones, o supuestos -que deben someterse a prueba (validarse)-, para explicar de mejor forma los acontecimientos y fenómenos de la vida económica con el fin de mejorar las

* Profesor de la Facultad de Economía, unAm y alumno de la Maestría en Ciencias en Metodología de la Ciencia, CIECAS-IPN. Agradezco a Francisco Castillo Cerdas, Hugo Contreras Sosa, Daniel E. Díaz Espinosa y especialmente a Sergio Hernández Castañeda y a Carlos A. López-Morales por sus valiosos comentarios para llegar a la versión final del documento aunque, como es normal, las ideas aquí expresadas son enteramente mi responsabilidad. 
predicciones (Friedman, 1953). ${ }^{1}$ Para el profesor de la Universidad de Chicago Robert Lucas, entonces, "somos básicamente narradores, creadores de sistemas económicos ficticios" (Lucas, 1988) y, dado el avance de la ciencia y del tipo de formalización matemática utilizada, se han desarrollado modelos que explican más fehacientemente la realidad y que les confieren de consistencia al quehacer de la profesión.

La modelación económica formal hace uso de herramientas matemáticas -desde las rudimentarias hasta las más sofisticadas- para explicar el comportamiento, las conexiones y la relación entre las variables exógenas y las endógenas (dependiendo de cada uno) de los modelos y de la teoría con las cuáles se construyen esos "sistemas paralelos o análogos" (Lucas, 1980). ${ }^{2}$ Esa interacción entre las variables, en el análisis económico, implica considerar nociones de equilibrio (también llamado estado de reposo, aparato metodológico, balance de fuerzas opuestas, etc.). Para Lucas "el equilibrio es sólo una forma en la que nosotros vemos las cosas, no es una propiedad de la realidad" (Snowdon y Vane, 2005). ${ }^{3}$ Un modelo que incluya $n$ variables relevantes, que tenga una consistencia lógica y que nos ofrezca un claro entendimiento del problema analizado, puede tener una enorme variedad de posiciones de equilibrio.

En ese marco antes expuesto, el objetivo del artículo es describir cuál es el vínculo entre el instrumental matemático y el equilibrio económico con la siguiente estructura: la primera sección describe una herramienta topológica, surgida de la geometría, llamada teorema de punto fijo: en particular se muestra en qué consisten los teoremas de Brouwer y de Kakutani, como inciso $a$, y una breve definición de equilibrio, como inciso $b$; en la segunda sección se revisará cómo se inserta en los equilibrios walrasiano y de Nash el bosquejo de los teoremas de puntos fijos de la sección anterior y, así, probar sus existencias; el documento cierra con algunas ideas que intentan mostrar la pertinencia de las dos secciones previas y un breve comentario general.

1 Este es uno de los puntos principales del debate sobre la cientificidad de la economía, el "realismo de los supuestos vs. potencia predictiva" (Contreras, 2013).

2 Aquí entran en la discusión dos métodos que permean en las ciencias sociales: el método naturalista -que busca conocer a una sociedad mediante abstracciones, necesarias, retomado los resultados más importantes de otras ciencias sociales, teniendo como hilo conductor los modelos surgidos de las ciencias de la naturaleza- y el método historicista -que intenta conocer a la sociedad "interpretando" solamente el sentido de la acción humana.

3 Esa fue la respuesta que Lucas le dio en Nueva Orleans a Brian Snowdon y a Howard Vane, profesores de las Universidades de Northumbria y Liverpool John Moores, respectivamente, en enero de 1997 al cuestionarlo sobre las críticas que habían tenido los modelos de equilibrio de los nuevos clásicos. 
Economía Informa núm. 388 septiembre - octubre • 2014 - " " " " " " " " " "

\section{Definiciones de puntos fijos y de equilibrio}

\section{A. Algunos elementos matemáticos de la teoría del equilibrio}

Los teoremas de punto fijo son algunas de las herramientas matemáticas básicas que se utilizan para demostrar la existencia de conceptos de solución, o de equilibrio, en la teoría económica. ¿Pero qué es un punto fijo? Puede definirse como sigue: sea $f$ una función ${ }^{4}$ del conjunto $K$ en él mismo. Un punto fijo de $f$ es un punto $z \in K$ que satisface $f(z)=z$. Dicho de otro modo, el punto fijo de una función (que asocia a cada elemento del dominio uno y sólo un elemento de la imagen) es un punto llamado $z$ que pertenece al conjunto $K \mathrm{y}$ cuya imagen bajo la función es el mismo $z$ (Border, 1985).

El primer teorema de punto fijo que se esbozará en esta sección es el teorema de Brouwer, en honor al matemático holandés Luitzen Egbertus Jan Brouwer (1881-1966). La versión más útil del teorema (Border, 1985) señala que:

Sea $K \subset \mathbb{R}^{n}$ un conjunto convexo ${ }^{5}$ y compacto $^{67}$ y sea $f: K \rightarrow K$ una función continua. ${ }^{8}$ Entonces $f$ tiene un punto fijo.

Pensemos en los siguientes dos ejemplos. Consideremos un círculo en el cual están insertos diversos puntos, $P$, que serán sometidos a una transformación, es decir, "una regla que asigna a todo punto $P$ otro punto $P$ ', llamado la imagen de $P$ bajo la transformación" (Courant y Robbins, 2010) y que, después de ella, habrá un punto que permanecerá en la misma posición. Ese punto será, entonces, un punto fijo (véase Gráfica 1, Panel A). Imaginemos ahora dos intervalos idénticos, $a$ y $b$, cuya función, $f(a)$ y $f(b)$, es convexa y compacta de $a$ y $b$ y que forma una curva (o gráfica) de identidad de $45^{\circ}$. El resultado de la función, al intersectarse con la curva de $45^{\circ}$, inducirá al menos un punto fijo, es decir, existirá algún punto $x^{*} \in(a, b)$ tal que $f\left(x^{*}\right)=x^{*}$ (véase Gráfica 1, Panel B).

4 Las funciones se denotan con una flecha $\rightarrow$.

5 “Un conjunto $K$ en $\mathbb{R}^{n}$ es convexo si $x, y \in K y \lambda \in[0,1] \Rightarrow \lambda x+(1-\lambda) y \in K$ ” (Berck y Sydsæter, 1994).

6 "Un conjunto $K$ en $\mathbb{R}^{n}$ es compacto (cerrado y acotado) si y sólo si toda sucesión de puntos de $K$ tiene una subsucesión que converge a un punto de K”' (Berck y Sydsæter, 1994).

7 Cuando hablamos de conjuntos convexos y compactos estamos hablando de conjuntos cerrados.

8 Decimos que una función $f$ es continua en $a$ si el $\lim _{x \rightarrow a} f(x)=f(a)$. 


\section{Gráfica I}

\section{Vectores de transformación y punto fijo}

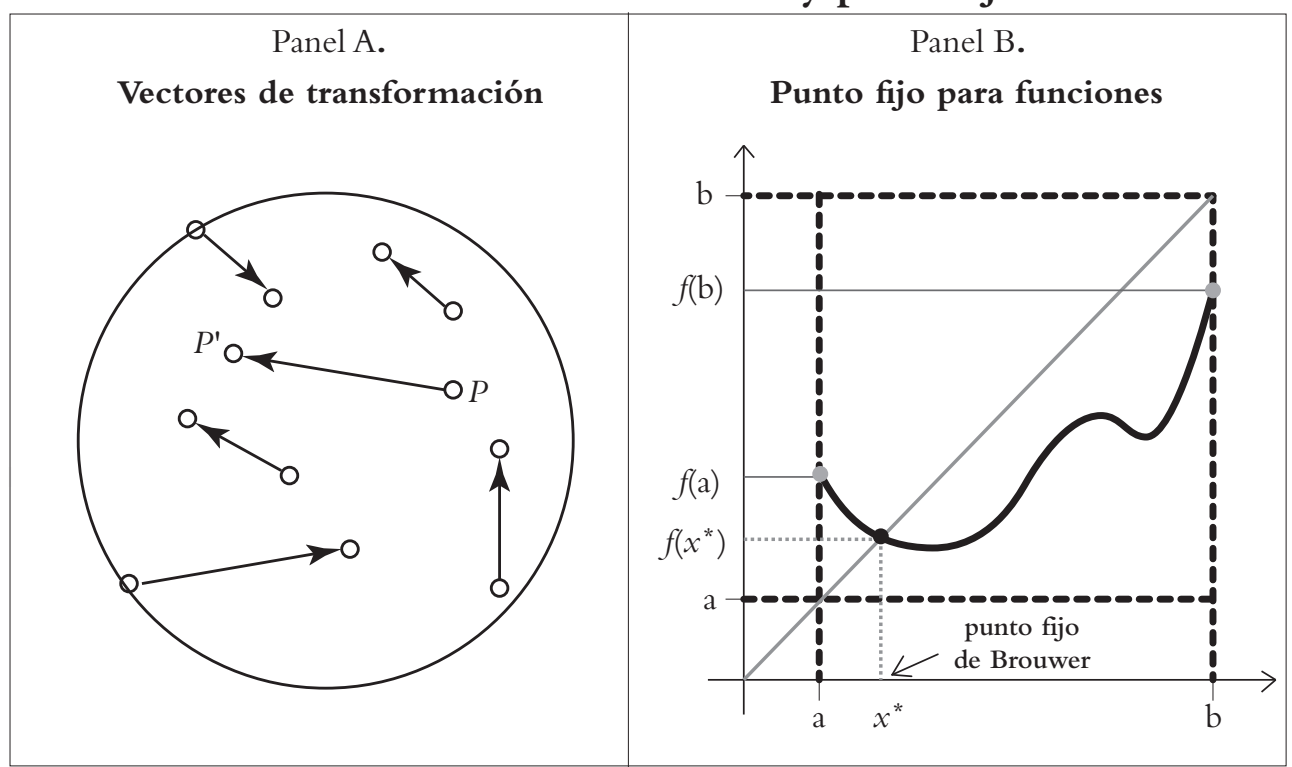

Fuente: tomado de Courant y Robbins, 2010 y elaboración propia, respectivamente.

El segundo teorema de punto fijo relevante puede plantearse como sigue: un punto fijo de una correspondencia ${ }^{9} \mu$ (que puede asociar varios elementos de la imagen a cada elemento del dominio) es un punto $x$ que satisface $x \in \mu(x)$. Uno de los teoremas más famosos para correspondencias es el planteado por el matemático japonés Shizuo Kakutani (1911-2004). En él, se establece que:

Sea $K \subset \mathbb{R}^{n}$ un conjunto convexo y compacto y $\mu: K \multimap K$ una correspondencia que asocia a cada punto $x$ perteneciente a $K$ un subconjunto cerrado $\mu(\mathrm{x})$ de $K$. Entonces $\mu$, si es superiormente semicontinua, tiene al menos un punto fijo.

Al igual que con los puntos fijos para funciones, pensemos en el siguiente ejemplo: imaginemos dos intervalos idénticos, $a$ y $b$, cuya correspondencia, $f(a)$ y $f(b)$, es convexa y compacta de $a$ y $b$ y que forma una curva (o gráfica) de identidad de $45^{\circ}$. El resultado de la función, al intersectarse también con la curva de $45^{\circ}$, inducirá al menos un punto fijo que ahora está inserto en una

9 Las correspondencias se denotan con el símbolo $\multimap$. 
región entre $f(a)$ y $f(b)$, es decir, un punto $x^{*} \in K$, tal que $x^{*} \in \mu\left(x^{*}\right)^{10}$ (véase Gráfica 2).

\section{Gráfica 2}

\section{Puntos fijos para correspondencias}

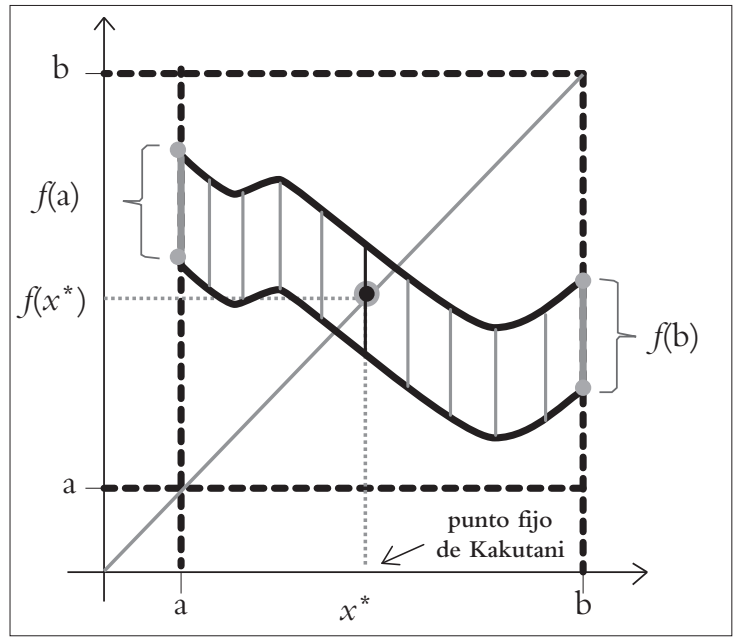

Fuente: elaboración propia.

En los distintos modelos matemáticos de la economía se puede sustituir el teorema de punto fijo de Brouwer por el teorema de punto fijo de Kakutani debido a que es más común definir correspondencias que valores únicos de una función. Es por ese motivo que el teorema de Kakutani "es una generalización del teorema de Brouwer que incluye correspondencias (de buen comportamiento) además de funciones" (Gibbons, 1993).

\section{B. El concepto de equilibrio}

¿Y qué es un equilibrio? A pesar de que en el análisis económico es un concepto común, no abundan las precisiones de manera concreta. Pero más que un concepto, el equilibrio -en primera instancia- debería entenderse como un método, un "equilibrio de fuerzas" que "se conecta con los modelos que contienen diversas variables interrelacionadas" (Machlup, 1958). El equilibrio puede entenderse, también, como "una idea central organizadora" (Hanh, 1984) o "un principio central alrededor del cual se organiza toda la teoría

10 Tomado, con algunas modificaciones ligeras, de Berck y Sydsæter, 1994. 
económica" (Milgate, 1987). Para Lucas (1980) el equilibrio significa una "optimización individual y una consistencia en los planes".

¿Pero cuál equilibrio es el más adecuado? Eso dependerá del contexto, los propósitos y los fines que se pretendan en las diferentes teorías. En economía existen diferentes tipos de equilibrios $-y$ por consiguiente diferentes tipos de modelos- que están acotados, quizá, a las diferentes metodologías que utiliza cada una de las escuelas de pensamiento. Están, por ejemplo, el equilibrio walrasiano (conocido como equilibrio general), el equilibrio de Nash (definido en la teoría de juegos no cooperativos), el equilibrio de expectativas racionales (equilibrio con incertidumbre), el equilibrio general restringido (modelos macro nuevo keynesianos de precios pegajosos) y los modelos de equilibrio general dinámico y estocástico, entre otros (Smith, 2013). ${ }^{11}$

Algunos economistas consideran que se "debería abandonar [la teoría del equilibrio] en favor de teorías que presten más atención a la historia" debido a que son "inapropiados para analizar a las economías en el mundo real” (Backhouse, 2004). Para los que se ciñen a la corriente principal de la profesión (mainstream), por lo general, la noción de equilibrio es "una característica ineludible" que se da por hecho en cualquier análisis económico. El análisis del equilibrio puede constar, básicamente, de cuatro pasos: 1) el modelo parte de una posición inicial de equilibrio; 2) con nuevos datos, el modelo se moverá de la posición inicial de equilibrio; 3 ) el modelo se ajusta ante el nuevo panorama y; 4) como resultado de la perturbación se arriba a una nueva posición de equilibrio distinta a la inicial (Machlup, 1958). A este tipo de equilibrios se les conoce como "parciales" o "estáticos".

Posiblemente uno de los modelos económicos más conocidos - planteado por Alfred Marshall- es el que relaciona la demanda (de los consumidores) con la oferta (de los productores) en un solo mercado -llamado equilibrio de "vaciado de mercado". En él, el precio se ajustará hasta que la cantidad demandada por los consumidores se iguale con la cantidad ofertada por las empresas y en ese punto el mercado estará en equilibrio parcial. Cuando se generaliza la noción de equilibrio marshaliano, los instrumentos matemáticos más apropiados para probar su existencia son los teoremas de punto fijo (véase Gráfica 3).

11 Para una visión más completa de los diferentes equilibrios véase Backhouse, 2004 y Smith, 2013. 


\section{Gráfica 3}

\section{Representación clásica del equilibrio}

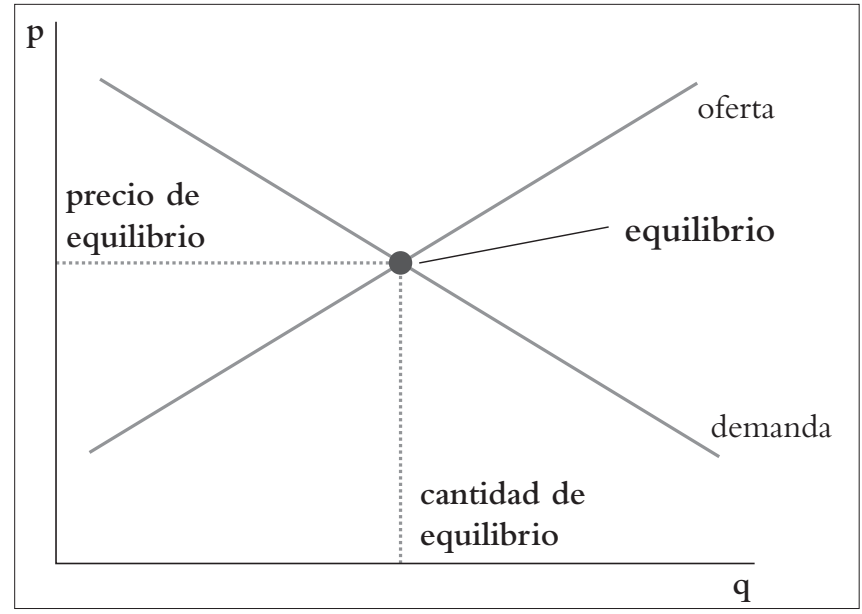

Fuente: elaboración propia.

\section{Equilibrio general' ${ }^{12}$ y equilibrio de Nash}

Un modelo que nos es útil para analizar el equilibrio "de manera relativamente simple" es el planteado en la famosa "caja de Edgeworth". ${ }^{13}$ En ella se muestra la interacción de dos agentes, digamos Lucas y Sargent, con dos bienes, $x$ y $y$, en una economía pequeña, sin producción, donde cualquier punto dentro de la caja es una asignación disponible de cada bien para los agentes. En el punto $E$ y en el punto $F$, tanto Lucas (que tiene mayor dotación del bien $X$ pero poco del bien $Y$ ) como Sargent (que tiene mayor dotación del bien $Y$ pero poco del bien $X)$ pueden, mediante el intercambio de sus dotaciones iniciales, moverse a un punto mutuamente beneficioso, caracterizado por los puntos de tangencia $M_{1}$, $M_{2}, M_{3}$ y $M_{4}$. En tales puntos no hay forma de que Lucas mejore su bienestar sin empeorar el de Sargent y la suma de esas asignaciones eficientes integran la llamada curva de contrato (es decir, la línea que cruza la caja del punto de origen de Lucas, $O_{L}$, al punto de origen de Sargent, $O_{S}$ ). Esa es la idea central de una asignación eficiente en el sentido de Pareto, o un equilibrio en el sentido de Pareto (véase Gráfica 4).

12 Cabe aclarar que en este apartado no se abordarán las críticas y posibles soluciones al equilibrio económico general. Los aportes de Abraham Wald, Kenneth Arrow y Gerard Debreu, por citar algunos, se expondrán someramente.

13 Llamada así en honor al economista británico Francis Ysidro Edgeworth. 


\section{Gráfica 4}

\section{Caja de Edgeworth}

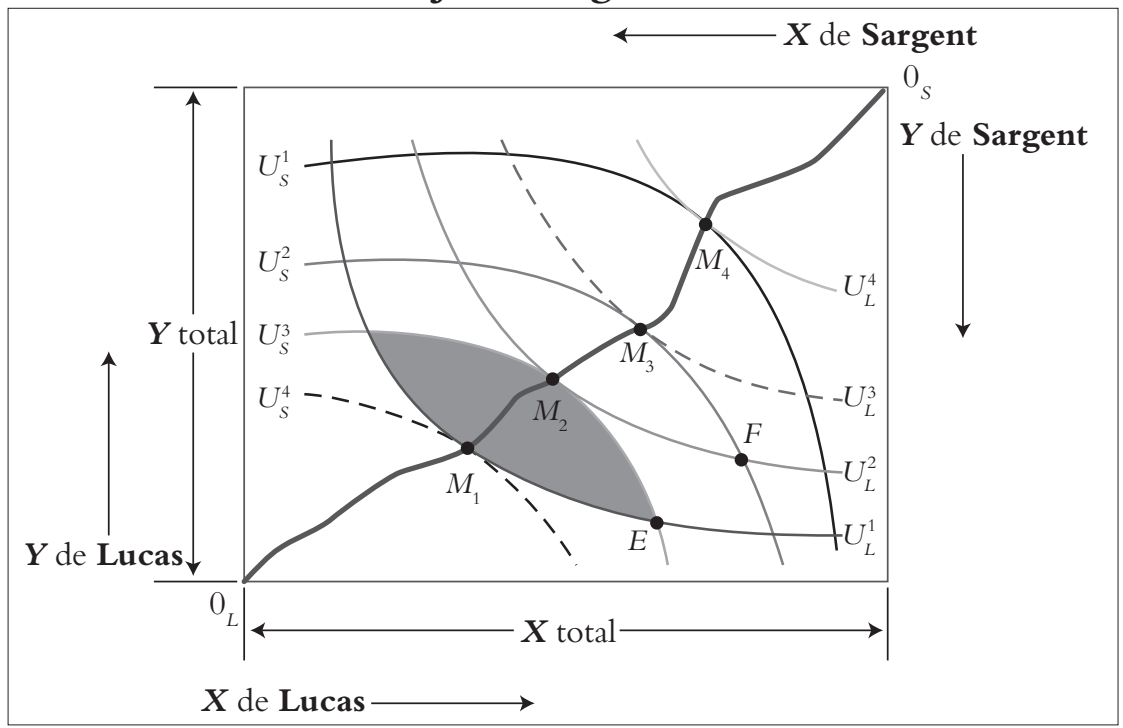

Fuente: tomado de Nicholson y Snyder, 2010.

El economista francés Léon Walras ${ }^{14}$ retomando la idea de que en un solo mercado la demanda es igual a la oferta, examinó en su libro Elementos de economía pura de 1874 el equilibrio general en todos los mercados en los que cada consumidor maximizaba su utilidad, o su riqueza, con sus dotaciones de bienes a los precios de mercado y cada productor maximizaba sus beneficios, o riqueza, con las acciones de su empresa respecto al precio de los factores. $\mathrm{La}$ solución de equilibrio propuesta por Walras consistía en el conteo del número de ecuaciones simultáneas de demanda y de oferta y el conteo del número de incógnitas, los precios y las cantidades. ${ }^{15}$

Formalmente, si tenemos $L$ bienes en el mercado, al consumidor, $i=1$, ..., I con un vector de consumo $X_{i} \subset \mathbb{R}^{L} \mathrm{y}$ un derecho, $\theta$, de las ganancias de la empresa, a la empresa, $j=1, \ldots, J$, con un conjunto de producción $Y_{i} \subset \mathbb{R}^{L}$, un

14 Considerado el fundador de la teoría matemática del equilibrio económico general.

15 Para el profesor emérito de la Universidad de Londres Mark Blaug "no está justificada por completo la creencia popular de que Walras se limitó a contar ecuaciones e incógnitas para demostrar la existencia de un equilibrio general de mercado. También intentó demostrar que el mercado resolvería las ecuaciones impulsando a la economía al equilibrio. Reconoció que no basta con demostrar la existencia de una solución de equilibrio sino que igualmente debe probarse que el equilibrio es estable "en lo pequeño" y "en lo grande", que es "determinado" en el sentido de que la posición final es independiente de la ruta que se siga hacia el equilibrio" (Blaug, 1990). 
Economía Informa núm. 388 septiembre - octubre • 2014 | " " " " " " " " " "

vector de recursos iniciales $\tilde{\omega}=\left(\tilde{w}_{1}, \ldots, \tilde{w}_{L}\right) \in \mathbb{R}^{L}$, asignaciones $(x, y)=\left(x_{1}, \ldots\right.$ $\left.x_{I}, y_{1}, \ldots, y_{J}\right) \in\left(X_{i}, Y_{i}\right)$, y un vector de precios $p=\left(p_{1}, \ldots, p_{L}\right)$, decimos que:

a) si para cada $\mathrm{j}, y_{j}^{*}$, maximiza sus ganancias en $y_{j}$; esto es:

$p \times y_{j} \leq p \times y_{j}^{*}$ para todo $y_{j} \in Y_{j}$

cada producto maximiza sus beneficios con los precios de equilibrio

b) si para cada $i, x_{j}^{*}$ es máxima para $\geqslant_{i}$ en el conjunto presupuestario:

$\left\{x_{i} \in X_{i}: p \times x_{i} \leq p \times \omega_{i}+\sum_{j} \theta_{i j} p \times y_{j}^{*}\right\}$

los consumidores maximizan su bienestar con los precios de equilibrio y con la riqueza derivada de la tenencia de bienes y de su participación en los beneficios

c) $\sum_{j} x_{i}=\tilde{\omega}+\sum_{j} y_{j}^{*}$

los mercados se vacían en equilibrio, es decir, no hay más comercio salvo en los precios de equilibrio (es una asignación eficiente en el sentido de Pareto) ${ }^{16}$

$a, b$ y constituyen las condiciones de un equilibrio walrasiano (o competitivo $)^{17}$

La solución de equilibrio propuesta por Walras no se quedó allí. El matemático rumano Abraham Wald fue el primero en mostrar explícitamente la solución matemática al sistema de ecuaciones e incógnitas propuestas por Walras aunque suponía muchas restricciones en las preferencias de los consumidores. Años más tarde, los economistas estadounidenses Kenneth Arrow y Lionel McKenzie, y también el economista francés Gerard Debreu, con base en la idea original de Wald, ofrecieron pruebas más detalladas sobre su existencia usando los teoremas de punto fijo. Estos trabajos son, quizá, los vínculos más fuertes y la razón fundamental por la cual es importante estudiar los teoremas de punto fijo y los diferentes equilibrios en economía.

Podemos exponer las ideas fundamentales para la prueba de la existencia del equilibrio económico general de la siguiente manera: en primer lugar, observemos que tanto la función de oferta, $O(P)=O\left(p_{1}, p_{2}, \ldots, p_{m}\right)$, como la de demanda, $D(P)=D\left(p_{1}, p_{2}, \ldots, p_{m}\right)$, son homogéneas de grado cero. Ello permite reducirnos solo a demostrar la existencia del equilibrio en el simplejo de dimensión $m-1, S_{m-1}=\left\{\left\langle P=\left(p_{1}, p_{2}, \ldots, p_{m}\right) \geq \overline{0} \mid \sum_{i=1}^{m} p_{i}=1\right\rangle\right\}$, un conjunto convexo y compacto sumergido en el espacio $\mathbb{R}^{m}$. Enseguida, con ayuda de la función de exceso de demanda $\Delta(P)=D(P)-O(P)$, definimos una nueva función

16 Asignación en la que no es posible mejorar el bienestar de ninguna persona sin empeorar el de alguna otra.

17 Tomado, con algunas modificaciones ligeras, de Mas-Colell et al., 1995. 
-inspirada por John Nash- T:S $S_{m-1} \rightarrow S_{m-1}$, donde $T(P)$ puede interpretarse como el nuevo vector de precios en el cual se transformaría $P$ si éste está sujeto a las fuerzas del mercado. Puede demostrarse entonces que $T$ es continua y, por lo tanto, en virtud del teorema de Brouwer, tiene al menos un punto fijo, $\dot{P}$. Ese punto resulta ser un equilibrio (Hernández, 2010).

Por otro lado, la teoría de juegos aplicada a la economía puede estudiarse desde varios ángulos. Los agentes económicos, que siempre tomarán las mejores decisiones para alcanzar sus objetivos, pueden llegar juntos a resultados óptimos cooperando en determinado juego. En situaciones más realistas los agentes, que utilizan toda la información que tienen disponible, actuarán guiados por su propio interés y por las posibles decisiones de los demás participantes del juego, aunque no siempre se llegue a resultados óptimos. ${ }^{18}$ Uno de los métodos para llegar al equilibrio en estos juegos no cooperativos fue planteado en 1949 por el matemático estadounidense John F. Nash y que ahora lleva su nombre, el llamado equilibrio de Nash.

Si representamos matemáticamente un juego estratégico, $G$, donde interactúan $n$ jugadores, denotados, cada uno, con la letra $i$, con estrategias, $s_{i}$ contenidas en un conjunto $S_{i}$, donde $s_{i} \in S_{i}$, y una función de ganancias $u_{i}$, decimos que:

cuando la estrategia pura (o mixta), $s_{A}$, del jugador $A$ es la mejor respuesta ${ }^{19}$ (que puede ser una función o una correspondencia) respecto a la estrategia de $B$ y la estrategia pura (o mixta), $S_{B}$, del jugador $B$ es la mejor respuesta (que puede ser una función o una correspondencia) respecto a la estrategia de $A$, ese conjunto de estrategias forman un equilibrio de Nash, es decir:

$$
\begin{aligned}
& u_{i}\left(s *{ }_{i}, \ldots S *{ }_{n}\right) \geq u_{i}\left(S_{i}, \ldots S_{n}\right) \\
& \text { donde } S * \text { es una solución de } \\
& \max _{\left(s_{i} \in S_{i}\right)} u_{i}\left(S *{ }_{i}, \ldots S *{ }_{n}\right)^{20}
\end{aligned}
$$

18 Este comportamiento de los agentes se semeja a las ideas del economista Adam Smith (17231790) plasmadas en su obra La riqueza de las naciones (1776) sobre el supuesto de la mano invisible, describiendo que el actuar de cada uno de los agentes en beneficio propio hacía que el sistema de mercado funcionara en beneficio de todos.

19 "La estrategia a del jugador $A$ es la mejor respuesta a la estrategia del jugador $B$ si $A$ no puede ganar más con ninguna otra posible estrategia dado que $B$ está jugando la estrategia $b$ " (Nicholson y Snyder, 2010).

20 Tomado, con algunos modificaciones ligeras, de Gibbons, 1993. 
Economía Informa núm. 388 septiembre - octubre • 2014 | " " " " " " " " " "

Ese equilibrio de Nash puede ejemplificarse, de manera sencilla, en el que probablemente sea el juego más famoso: el dilema del prisionero. ${ }^{21}$ La policía detiene a dos presuntos ladrones y los someten a interrogatorios por separado; los delincuentes, sin haber tenido oportunidad de ponerse de acuerdo de lo que responderán, se encuentran ante las siguientes opciones:

- No confesar y quedar en libertad si el otro toma la misma decisión

- Confesar y quedar en libertad si el otro no confiesa

- No confesar y ser castigado si el otro confiesa

- Ambos confesarán y pagarán una condena menor

El problema estriba en que cada una de las partes tiene incentivos para confesar en la medida en que espera que el otro prisionero haga lo propio. ${ }^{22} \mathrm{Si}$ el prisionero $A$ decide no confesar y $B$ lo delata el prisionero $A$ tendrá que pagar una condena de cuatro años, mientras que su cómplice saldrá libre; lo mismo ocurre para el caso inverso donde $A$ confiesa y $B$ no lo hace. Por otra parte, si los dos prisioneros deciden no confesar pasarían un año encarcelados; sin embargo, existen incentivos para cambiar la decisión en ese punto, debido a que cambiarla implicaría obtener su libertad (véase Cuadro 1).

\section{Cuadro I}

\section{Matriz de resultados: el dilema del prisionero}

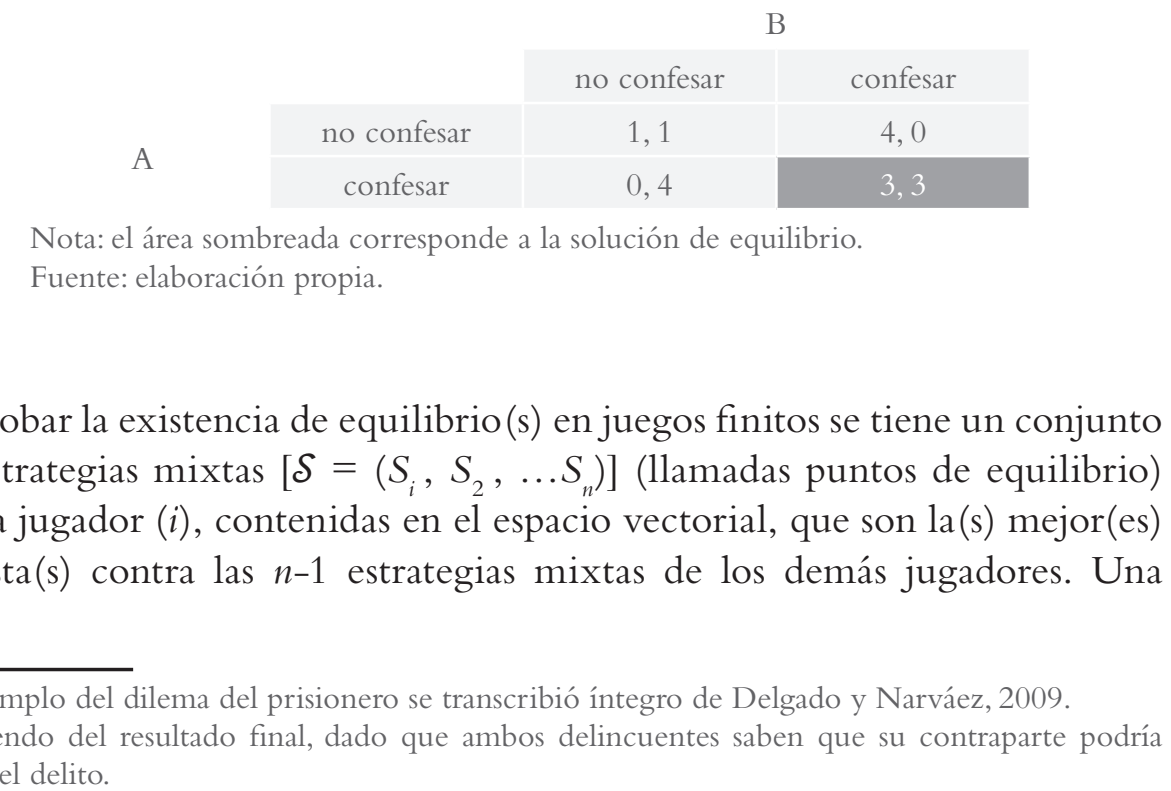

22 Partiendo del resultado final, dado que ambos delincuentes saben que su contraparte podría confesar el delito. 
combinación de ese conjunto de estrategias es un equilibrio de Nash, es decir, el mapeo tiene un punto fijo según el teorema de Kakutani, si y sólo si:

$$
p_{i}(\mathcal{S})=\max _{\text {todas las } r_{i}^{\prime} \text { 's }}\left[p_{i}\left(\mathcal{S} ; r_{i}\right)\right]
$$

Entonces, "el punto de equilibrio es un conjunto $\mathcal{S}$ donde cada estrategia mixta óptima de los jugadores maximiza su función de pago, $p_{i}(\mathcal{S})$, si las estrategias de los otros se mantienen fijas" (Nash, 1951). Para probar la existencia de equilibrio(s) en juegos suponemos que "cualquier punto fijo de una cierta correspondencia [o función] es un equilibrio de Nash y se demuestra que esa correspondencia [o función], con el teorema de punto fijo más adecuado [que puede ser, por ejemplo, el de Brouwer o Kakutani], debe tener un punto fijo" (Gibbons, 1993).

\section{Comentarios finales}

Los resultados mencionados en las secciones previas, empezando con el fundador de la teoría matemática del equilibrio general León Walras (1834-1910) y el "primer modelo multiecuacional para el equilibrio de oferta y demanda en todos los mercados simultáneamente" (Sydsæter et al., 2012) en un sistema de mercado perfectamente competitivo, y desarrollados más ampliamente con las ideas de otro grupo de economistas sobre equilibrio general, ya en el siglo xx, serían premiadas con el Nobel de Economía: a Kenneth J. Arrow (1921...) y a John R. Hicks (1904-1989) en 1972 por "su pionera contribución a la teoría del equilibrio económico general y a la teoría del bienestar"; a Gerard Debreu (1921-2004) en 1983 por "haber incorporado nuevos métodos analíticos en la teoría económica y por su rigurosa reformulación de la teoría del equilibrio general"; y a John C. Harsanyi (1920-2000), John F. Nash (1928...) y Reinhard Selten (1930-...) en 1994 "por su análisis pionero del equilibrio en la teoría de los juegos no cooperativos”, según lo expuesto por la Real Academia Sueca de las Ciencias.

Las aportaciones que diversos autores, además de los mencionados, hicieron a la noción de equilibrio se potenciaron, sin lugar a dudas, por el uso de herramientas matemáticas - como los teoremas de punto fijo- cada vez más sofisticadas que han permitido verificar, sólidamente, hipótesis económicas con el incremento de evidencias empíricas. Ese desarrollo de las técnicas de modelación se ha extendido, casi a la par, con nuevos supuestos en la profesión, que han revolucionado nuestra manera de estudiar economía (como la 
Economía Informa núm. 388 septiembre - octubre • 2014 | " " " " " " " " - "

hipótesis de las expectativas racionales). A pesar de que en ese transitar están insertos innumerables debates entre las escuelas de pensamiento, lo cierto es que cada uno de ellos ha puesto una piedra más hasta llegar al conocimiento estándar de la economía moderna (Blaug, 1990).

Se ha descrito brevemente en qué consisten los teoremas de punto fijo de Brouwer y de Kakutani, en qué consiste un equilibrio y cómo se insertan esas nociones para probar la existencia de dos equilibrios: el general y el de Nash. Esa relación interdisciplinar entre las matemáticas y la economía evidencia, una vez más, que los economistas no pueden ser ajenos al conocimiento de métodos matemáticos diversos para poder introducirse, por ejemplo, a la economía estándar -que hoy domina en diversas licenciaturas y posgrados en economía a nivel mundial. Y a nivel nacional las cosas son muy distintas. Todavía hay quienes, aún con el estado del arte de la ciencia económica, afirman falsamente algo que el británico Ian Stewart (2006) ya consideraba, que “...la matemática carece de utilidad, que no importa, que sólo son juegos intelectuales sin un verdadero significado... que sólo son números, ecuaciones, leyes o fórmulas".

\section{Bibliografía}

Backhouse, Roger (2004), "History and equilibrium: a partial defense of equilibrium economics", Journal of Economic Methodology vol. 11 núm. 3, mayo.

Berck, Peter y Knut Sydsæter (1994), Formulario para economistas, Ed.Antoni Bosch, España.

Blaug, Mark (1990), Economic theory in retrospect, Cambridge University Press, Cambridge.

Border, Kim (1985), Fixed point theorems with applications to economics and game theory, Cambridge University Press, Cambridge.

Contreras, Hugo (2013), “Centralidad analítica del equilibrio general”, documento de trabajo del Seminario de Credibilidad Macroeconómica, FE-UnAm, México.

Courant, Richard y Herbert Robbins (2010), ¿Qué son las matemáticas? Conceptos y métodos fundamentales, Ed. Fondo de Cultura Económica, México.

Delgado, Gabriel y René Narváez (2009), "Estados Unidos: reformando al sistema de salud sin cooperación”, Economía Informa núm. 361, FE-UNAM, noviembrediciembre.

Friedman, Milton (1953), "Methodology in positive economics" en Essays in positive economics, The University of Chicago Press, Chicago.

Gibbons, Robert (1993), Un primer curso de teoría de juegos, Ed. Antoni Bosch, España. 
Hahn, Frank (1984), Equilibrium and Macroeconomics, MIT Press, Cambridge.

Hernández, Sergio (2010), "Una clase de mercados competitivos simplificados", no publicado, marzo.

Lucas, Robert (1980), "Methods and problems in business cycle theory", Journal of Money, Credit and Banking vol. 12 núm. 4, noviembre.

--- (1988)/ "What economist do", unpublished, diciembre.

Machlup, Fritz (1958), "Equilibrium and disequilibrium: misplaced concreteness and disguised politics", The Economic Journal vol. 68 núm. 269, marzo.

Mankiw, Gregory (2013), Macroeconomics, Worth Publishers, Nueva York.

Mas-Colell, Andreu, Michael Whinston y Jerry Green (1995), Microeconomic theory, Oxford University Press, Nueva York.

Milgate, Murray (1979), 'On the origin of the notion of "intertemporal equilibrium”, Economica vol. 46 núm. 181, febrero.

Nash, John (1950), "Equilibrium points in n-person games", Proceedings of the $\mathrm{Na}$ tional Academy of Sciences of the United States of America vol. 36 núm. 1, enero.

--- (1951), "Non-cooperative games", The Annals of Mathematics vol. 54 núm. 2, septiembre.

Nicholson, Walter y Christopher Snyder (2010), Intermediate microeconomics and its applications, South-Western, Cengage Learning, Ohio.

Snowdon, Brian y Howard R. Vane (2005), Modern Macroeconomics. Its Origins, Development and Current State, Edward Elgar Publishing, Massachusetts.

Smith, Noah (2013), "What is an economic equilibrium?”, Noahpinion, abril.

Spivak, Michael (1996), Cálculo infinitesimal, Ed. Reverté, México.

Stewart, Ian (2006), Letters to a young mathematician, Basic Books, Nueva York.

Sydsæter, Knut, Peter Hammond y Andrés Carbajal (2012), Matemáticas para el análisis económico, Ed. Pearson Educación, Madrid. 